\title{
Early experience with four-dimensional computed tomography for observing velopharyngeal closure function in pediatric patients and the evaluation of the exposure dose
}

Yoshikazu Kobayashi ( $\nabla$ y.kobayashi.oms@gmail.com )

Fujita Hoken Eisei Daigaku https://orcid.org/0000-0003-1416-7066

Masanao Kobayashi

Fujita Hoken Eisei Daigaku

Daisuke Kanamori

Fujita Hoken Eisei Daigaku

Naoko Fujii

Fujita Hoken Eisei Daigaku

Yumi Kataoka

Fujita Hoken Eisei Daigaku Byoin

Koji Satoh

Fujita Hoken Eisei Daigaku

Yoshimi Sano

Fujita Hoken Eisei Daigaku

Satoshi Yoshioka

Fujita Hoken Eisei Daigaku

Ichiro Tateya

Fujita Hoken Eisei Daigaku

Hiroshi Toyama

Fujita Hoken Eisei Daigaku

Koichiro Matsuo

Fujita Hoken Eisei Daigaku

Research article

Keywords: velopharyngeal closure, Monte Carlo simulation, exposure dose, four-dimensional computed tomography, hypernasality

Posted Date: July 16th, 2020

DOI: https://doi.org/10.21203/rs.3.rs-37116/v1 
License: () (1) This work is licensed under a Creative Commons Attribution 4.0 International License. Read Full License 


\section{Abstract \\ Background}

The aims of this study were to perform a four-dimensional assessment of velopharyngeal closure function in pediatric patients with cleft palate using 320-row area detector computed tomography (CT), and to estimate the organ-absorbed doses using Monte Carlo simulation.

\section{Methods}

We evaluated CT image data obtained between July 2018 and August 2019 from five pediatric patients with cleft palate (four boys and one girl; age range, 4-7 years) at Fujita Health University Hospital. The presence of velopharyngeal insufficiency (VPI), patterns of velopharyngeal closure (VPC), and cross-sectional area of VPI were evaluated. In addition, organ-absorbed doses were assumed in the Monte Carlo simulation. However, we did not perform statistical analysis because of the insufficient number of patients enrolled in this study.

\section{Results}

The existence of VPI and hypernasality were completely concordant. The VPC patterns were circular (two patients), circular with Passavant's ridge (one patient), and unevaluable (two patients). The organ-absorbed doses were relatively lower than those in past reports.

\section{Conclusions}

Our method could be an alternative for patients who refuse the conventional nasopharyngoscopic evaluation.

\section{Background}

Anatomical abnormalities of the nasopharyngeal space that are associated with cleft palate result in velopharyngeal insufficiency (VPI), which may cause dysarthria and dysphagia [1]. If VPI remains despite attempts to improve function with surgery and speech pathology evaluation, a secondary surgery is indicated to achieve function. Various examinations are used to assess velopharyngeal function (VPF) to determine the surgical or nonsurgical approach to improve VPF. The gold standard for assessing VPF is a speech pathology evaluation by a speech-language pathologists (SLPs). However, to determine the application of a secondary surgery and the choice of the procedure, an examination of anatomical abnormalities is necessary. Two main areas of dysfunction are (1) between the soft palate and posterior pharyngeal wall and (2) the lateral walls of the pharynx. Nasopharyngoscopy is the only procedure that allows the observation of both areas; however, it requires the insertion of an endoscope, which may result in much discomfort that often makes an examination difficult to perform. 
In 2019, we reported a four-dimensional (4D) evaluation method that appends the time phase image to threedimensional (3D) images for the assessment of velopharyngeal closure (VPC) by using 320-row area detector computed tomography (320-ADCT) images acquired from adult volunteers; this method was named "virtual nasopharyngoscopy" [2]. When using this method, the time and dose of exposure were reduced, compared to those of a previously reported method [3]. In this study, we report the application of our method to pediatric patients and estimate the organ-absorbed doses in these patients by using Monte Carlo (MC) simulation.

\section{Methods}

\section{Patients}

We evaluated the computed tomography (CT) image data of five pediatric patients (four boys and one girl; age range, 4-7 years); the CT images were obtained between July 2018 and August 2019. The participants consisted of three preoperative patients with submucosal cleft palate and two postoperative patients with unilateral cleft lip and palate. All participants had received dysarthria training for more than 6 months; however, they all presented with persistent hypernasality in speech pathology evaluations by SLPs. In addition, nasopharyngoscopic evaluations could not be performed in these patients (except for two patients) because of persistent crying. The study was conducted in accordance with the Declaration of Helsinki (1964) and was approved by the Fujita Health University Ethics Review Committee (Aichi, Japan; reference number: HM18-431). All parents or legal guardians of the participants were sufficiently informed about the purpose of this study, and they provided written informed consent for their child's personal or clinical details to be published in this study.

\section{Imaging procedure}

CT imaging using a 320-ADCT scanner (Aquilion ONE/Genesis Edition; Toshiba Medical Systems Corp., Tochigi, Japan) was conducted, using the method described in our previous report [2]. Each participant lay horizontally on the bed, and performed two successive tasks following the signal of the examiner: nasal inspiration, followed by oral expiration through a catheter into a water-filled cup. Scanning began immediately after the onset of inspiration. Expiration continued 1 second after starting and until the end of the scanning. We used a 14-French gauge catheter ( $4.7 \mathrm{~mm}$ outer diameter, $47 \mathrm{~cm}$ long; Nurvie; Covidien Japan, Tokyo, Japan) and water-filled disposable clear polyethylene terephthalate cups (370 mL) (Fig. 1). The scanning parameters were set as follows: field of view (FOV), $240 \mathrm{~mm}$; scanning length, 80-120 mm; tube voltage, $120 \mathrm{kV}$; tube current, $30 \mathrm{~mA}$; and exposure time, $3.30 \mathrm{~s}$ ( $0.275 \mathrm{~s} /$ rotation $\times 12$ rotations). For each child, the scanning length was set between the inferior border of the mandible and the infraorbital border to reduce exposure to the thyroid gland and eyeball. Two patients performed the tasks twice because they failed to execute the protocol. To improve temporal resolution, we implemented a half-reconstruction technique by which images were generated from 0.138 -second data for image generation. The medians of the volume of the CT dose index (CTDI ${ }_{\mathrm{vol}}$ ) and the dose length product (DLP) for each scan were $1.02 \mathrm{mGy}$ $(12.20 \mathrm{mGy} / 12$ rotations) and $119.9 \mathrm{mGy} \times \mathrm{cm}$, respectively.

\section{Image analysis}


The images were drawn with 0.5 -mm slice thickness and transferred to a medical imaging workstation (Ziostation2; Ziosoft, Inc., Tokyo, Japan), where 4D images of airway mobility (i.e., airway-mode), 4D images in virtual endoscopy mode (Additional file 1, Video 1), and multiplanar reconstruction (MPR) images were generated (Fig. 2). The airway-mode, virtual endoscopy mode, and MPR images had a window length of 500 $\mathrm{HU}, 75 \mathrm{HU}$, and $40 \mathrm{HU}$, respectively, and a window width of $500 \mathrm{HU}, 500 \mathrm{HU}$, and $400 \mathrm{HU}$, respectively. The movement of the velopharyngeal structures was recorded during the examination, and the presence of VPI and VPC patterns were estimated. The VPC patterns were categorized into four groups: coronal, sagittal, circular, and circular with Passavant's ridge, based on earlier reports [4] (Fig. 3). The minimum crosssectional area (CSA) of the nasopharynx was measured parallel to the palatal plane, using the MPR images obtained during blowing.

\section{Exposure dose calculation}

Based on the protocol described in a previous report [5], we estimated organ-absorbed doses by using MC simulation with ImpactMC (CT imaging $\mathrm{GmbH}$, Erlangen, Germany). With this software, the air kerma, bowtie filter shape data, X-ray spectrum data, CT-to-density conversion coefficients, and scanning conditions of Digital Imaging and Communications in Medicine (DICOM) images can be input to calculate the absorbed doses for individual voxels while taking into account the photon interaction process. Air kerma for 4D-CT imaging was measured with an ionization chamber dosimeter (Model 9015; Radcal, Monrovia, CA, USA) by placing a pencil-type ionization chamber dosimeter (10X-3CT; Radcal, Monrovia, CA, USA) at the isocenter of the X-ray CT apparatus. The shape of the bow-tie filter in the X-ray CT apparatus was evaluated, based on Xray attenuation.

In the present study, the bow-tie filter shape was determined by measuring air kerma by moving the penciltype ionization chamber along the X-axis for $180 \mathrm{~mm}$ in 10-mm intervals (Fig. 4). Directly measuring the primary energy spectrum of the X-ray CT apparatus is difficult because of the structure of the system. Therefore, a carbon rod was placed at the isocenter, and the energy spectrum of scattered radiation scattered at $90^{\circ}$ was measured using a spectrum analyzer (RAMTEC413; Toyo Medic Co., Ltd., Tokyo, Japan) and a detector (XR-100T-CdTe; Amptek, Bedford, MA, USA) (Fig. 5). The energy spectrum of the scattered radiation was converted to the primary ray energy spectrum, based on the Klein-Nishina formula. A software standard conversion table was used, based on the CT-to-density conversion coefficients of water $\left(0 \mathrm{HU}: 1 \mathrm{~g} / \mathrm{cm}^{2}\right)$ and air (-1000 HU: $0 \mathrm{~g} / \mathrm{cm}^{2}$ ) (Fig. 6). MC simulation was conducted by inputting the scanning conditions of the DICOM images from 4D-CT imaging and other required items (Table 1) to obtain the absorbed dose distributions (Fig. 7). For each patient's absorbed dose distribution, 5-pixel square regions of interest (ROIs) were randomly selected at five locations in each target organ (i.e., brain, tongue, vocal cords, esophagus, thyroid, nasopharynx, oropharynx, hypopharynx, crystalline, and salivary gland) to evaluate each of the organ-absorbed doses.

Table 1. The Monte Carlo simulation condition 


\begin{tabular}{|ll|}
\hline Air kerma (mGy/100 mAs) & 17.47 \\
\hline Average tube current $(\mathrm{mA})$ & 30 \\
\hline Focus-center of rotation distance $(\mathrm{mm})$ & 600 \\
\hline Fan angle (rad) & 0.9 \\
\hline Rotation time (s/rot) & 0.275 \\
\hline Number of rotations (rot) & 12 \\
\hline Total beam collimation $(\mathrm{mm})$ & $80 / 100 / 120$ \\
\hline
\end{tabular}

\section{Statistical analysis}

We were unable to perform statistical analysis because of the insufficient number of patients enrolled in this study.

\section{Results}

\section{Image analysis}

The CT images revealed VPI in all patients (Table 2). In the patients' assessment, VPI and hypernasality were completely concordant. The VPC patterns were evaluated as circular (two patients) and circular with Passavant's ridge (one patient); the other patterns (two patients) were unevaluable because of poor mobility of the structures in the velopharyngeal spaces. While blowing, some participants sealed the nostril with the upper lip or blew air that was stored in the oral vestibule (Fig. 8). The CSA of VPI ranged $3.42-271 \mathrm{~mm}^{2}$.

Table 2

Distribution of velopharyngeal insufficiency and patterns of velopharyngeal closure

\begin{tabular}{|c|c|c|c|c|c|c|c|c|c|c|}
\hline \multirow{2}{*}{$\begin{array}{l}\text { Patient } \\
\text { no. }\end{array}$} & \multicolumn{2}{|c|}{ Age } & \multirow[t]{2}{*}{ Sex } & \multirow{2}{*}{$\begin{array}{l}\text { Cleft } \\
\text { type }\end{array}$} & \multirow[t]{2}{*}{ Status } & \multirow[t]{2}{*}{ VPI } & \multirow[t]{2}{*}{ VPC pattern } & \multirow{2}{*}{$\begin{array}{l}\text { Cross- } \\
\text { sectional } \\
\text { area of } \\
\text { VPI } \\
\left(\mathrm{mm}^{2}\right)\end{array}$} & \multirow{2}{*}{$\begin{array}{l}\text { CTDI }_{\text {vol }} \\
\text { (mGy) }\end{array}$} & \multirow{2}{*}{$\begin{array}{l}\text { DLP } \\
(\mathrm{mGy} \\
\times \mathrm{cm})\end{array}$} \\
\hline & $y$ & mo. & & & & & & & & \\
\hline$\# 1$ & 7 & 0 & M & SMCP & Primary & + & Unevaluable & 238 & 13.1 & 174.5 \\
\hline \multirow[t]{2}{*}{ \#2 } & 4 & 2 & $F$ & SMCP & Primary & + & $\begin{array}{l}\text { Circular } \\
\text { with }\end{array}$ & 3.42 & 12.5 & 150.5 \\
\hline & & & & & & & $\begin{array}{l}\text { Passavant's } \\
\text { ridge }\end{array}$ & & & \\
\hline \#3 & 5 & 5 & M & UCLP & Secondary & + & Circular & 15.79 & 11.9 & 119.9 \\
\hline$\# 4$ & 5 & 4 & M & UCLP & Secondary & + & Unevaluable & 161 & 12.2 & 97 \\
\hline \#5 & 4 & 10 & $M$ & SMCP & Primary & + & Circular & 271 & 12 & 119.2 \\
\hline
\end{tabular}


$\mathrm{CTDI}_{\mathrm{vol}}$, volume of the computed tomography dose index; DLP, dose length product; F, female; M, male; mo., months; Primary, primary operation; Secondary, secondary operation; SMCP, submucosal cleft palate; UCLP, unilateral cleft lip and palate; VPC, velopharyngeal closure; VPI, velopharyngeal insufficiency; $y$, years Exposure dose calculation

The absorbed doses of each organ are shown in Table 3. The scanning length was set between the inferior border of the mandible and the infraorbital border; however, radiation exposure to the thyroid gland and eyeball occurred in some patients because of body movement during imaging. Each absorbed dose was nevertheless relatively lower than the dose reported in past reports in which swallowing was evaluated with CT examinations in adult patients $[5,6]$.

Table 3. The calculated organ-absorbed doses of each patient

\begin{tabular}{|lllllll|}
\hline \multirow{5}{*}{ Organ } & \multicolumn{2}{l}{ Patient no. } & & & & $\begin{array}{l}\text { Median } \\
\text { [interquartile range] }\end{array}$ \\
\cline { 2 - 7 } & $\# 1$ & $\# 2$ & $\# 3$ & $\# 4$ & $\# 5$ & \\
\hline Brain & 6.64 & 6.66 & 6.68 & 7.97 & 7.84 & 6.68 [6.65-7.908] \\
\hline Tongue & 12.26 & 9.55 & 10.91 & 10.93 & 10.22 & 10.91 [9.89-11.60] \\
\hline Vocal cord & 11.91 & 9.56 & 10.11 & 10.13 & 13.03 & $10.13[9.83-12.47]$ \\
\hline Esophagus & 10.45 & $\mathrm{n} / \mathrm{a}$ & $\mathrm{n} / \mathrm{a}$ & $\mathrm{n} / \mathrm{a}$ & $\mathrm{n} / \mathrm{a}$ & 10.45 \\
\hline Thyroid gland & 10.31 & $\mathrm{n} / \mathrm{a}$ & $\mathrm{n} / \mathrm{a}$ & $\mathrm{n} / \mathrm{a}$ & $\mathrm{n} / \mathrm{a}$ & 10.31 \\
\hline Nasopharynx & 11.47 & 7.58 & 7.58 & 9.17 & 7.57 & $7.58[7.58-10.32]$ \\
\hline Oropharynx & 11.62 & 8.46 & 9.72 & 10.30 & 10.08 & 10.08 [9.09-10.96] \\
\hline Hypopharynx & 11.61 & 8.49 & 10.05 & 10.40 & 12.43 & 10.40 [9.27-12.02] \\
\hline Crystalline & 12.98 & $\mathrm{n} / \mathrm{a}$ & $\mathrm{n} / \mathrm{a}$ & 12.04 & $\mathrm{n} / \mathrm{a}$ & 12.51 \\
\hline Salivary gland & 12.91 & 10.76 & 10.89 & 11.67 & 10.97 & 10.97 [10.83-12.29] \\
\hline
\end{tabular}

The data are presented in mGy

n/a, not applicable

\section{Discussion}

In this study, we evaluated the reliability of "virtual nasopharyngoscopy," for assessing VPF in pediatric patients with cleft palate. We compared the findings of 320-ADCT versus those of the speech pathology evaluation. In general, the gold standard for estimating VPF is the detection of hypernasality in a speech pathology evaluation by a SLP. However, an imaging examination is essential for choosing the appropriate plan for treating VPI. Fiberoptic nasopharyngoscopy, which can anatomically identify the region and severity of VPI with 3D images, has an important role; however, it may cause pain, distress, or the vomiting reflex. 
Thus, pediatric patients often refuse to undergo this examination or fail to show sufficient ability to undergo an examination. Hence, an incomplete treatment plan or an inadequate surgical procedure for VPI may result.

In their 2015 study, Sakamoto et al. [3] reported the first 4D assessment of VPF in a patient with cleft palate by using 320-ADCT. They evaluated VPF in five pediatric patients during an exposure time of approximately 10 seconds and concluded that 4D-CT produces clear and detailed images with less stress and pain; it also had a shorter examination duration than that of other imaging modalities, and allows a quantitative VPC evaluation [3]. However, they reported that a major drawback of their procedure was an average radiation exposure of $4.44 \mathrm{mSv}$, which is larger than the level required in cephalometry, videofluoroscopy, and conventional CT [3].

In 2019, we reported a modified scan protocol that shortened the exposure time during VPF assessment [2]. In that study, we evaluated 10 adult volunteers, which included five patients with postoperative cleft. We concluded that nasopharyngoscopy and 4D evaluation with 320-ADCT had a high concordance rate for evaluating VPI (8/10 patients) and VPC patterns (9/10 patients) [2].

In the current study, we evaluated five pediatric patients with hypernasality. The presence of hypernasality and VPI in the CT images were completely concordant. The VPC patterns were evaluated as circular and circular with Passavant's ridge; however, two patients had unevaluable images. This finding was similar to the assessment of adult postoperative cleft patients reported in our past study: four patients had the circular pattern and one patient had the circular with Passavant's ridge pattern [2]. However, some participants in the current study demonstrated compensatory behavior during blowing to overcome the malfunction. This behavior was not observed in our past study, which assessed patients without VPI. Therefore, preparing an alternative task (e.g., sustained phonation) may be important when evaluating patients with VPI.

The CSA among patients with VPI ranged widely and was not associated with cleft type or whether a primary or secondary operation had been scheduled. We reported that the CSA among postoperative adult patients with VPI was at most $16.28 \mathrm{~mm}^{2}$ and they did not present with hypernasality. However, Patients \#2 and \#3 in this study had a smaller CSA and presented with hypernasality. The difference in CSA size among the same symptomatic patients remains unclear; however, future research will hopefully clarify this point.

In radiographic imaging of pediatric patients, the most concerning problem is exposure dose. Several investigators have estimated VPI or VPF by using dynamic magnetic resonance imaging $[7,8]$.This is a noninvasive technique that can be repeated without the risk of radiation exposure; however, compared to CT, magnetic resonance imaging's low imaging speed, narrow spatial coverage, and noise may interfere with the examination of pediatric patients.

We calculated the organ-absorbed dose with MC simulation to assess the radiation exposure in this examination as another goal in this study. Three types of dose estimation methods primarily exist: the use of an anthropomorphic phantom, a simulated reference man [9], or actual clinical images with MC simulation. Each method has advantages and disadvantages. The strongest advantage of MC simulation is the arbitrariness in setting the ROI size and place. We could then evaluate the details of the organ-absorbed dose 
by using MC simulation. However, this simulation cannot be used to assess the effective dose because of the lack of exposure dosage data outside of the coverage area.

The International Commission on Radiological Protection (ICRP) and many other international organizations have cooperated to optimize medical exposure. The ICRP recommended using the diagnostic reference level (DRL) to propel optimizing protection in the radiological diagnosis in its ICRP Publication 73 [10]. As mentioned in ICRP Publication 135 [11], DRLs are individually established in each country or region because equipment and procedure protocols can vary between different facilities. In general, the CTDI ${ }_{\text {vol }}$ or the DLP is described as the DRL. The CTDI ${ }_{\mathrm{vol}}$ is used to evaluate the performance of CT apparatuses. In 2015, the Japan Network for Research and Information on Medical Exposure published the report Diagnostic Reference Levels Based on Latest Surveys in Japan [12]. In the report, DRLs for pediatric head CT in children 1-5 years and 6-10 years were set at $47 \mathrm{mGy} \mathrm{CTDI}_{\text {vol }}$ and $60 \mathrm{mGy} \mathrm{CTDI}_{\text {vol, }}$, respectively. In our study, the CTDI $_{\text {vol }}$ of 320-ADCT was $12.20 \mathrm{mGy} / 12$ rotations. The coverage area does not correspond to the head completely; however, we believe that this amount of exposure is sufficiently optimized.

This study has some limitations. First, the short-duration protocol limits the number of feasible tasks. Therefore, the obtained results may not sufficiently reflect the actual VPI or hypernasality. In particular, the difference in the size of VPI or the influence of gravity is not sufficiently explained. In some patients, alternative procedures such as sustained pronunciation should be considered rather than blowing.

Second, the exposure dose remained much higher than that of conventional radiographic inspections. We hope future technological innovations will enhance the sensitivity of detectors and reduce the exposure dose. However, we believe that this amount of radiation exposure is acceptable with proper use because much information can be acquired with this method.

The greatest advantages of CT evaluation, compared to nonirradiating methods, are reproducibility, standardization, and the ability to allow for quantitative analysis. In this study, we did not prove the reproducibility of this method in the single examination of each participant. However, in future research, we will accumulate a number of cases and improve the reliability of our method.

\section{Conclusions}

We reported the clinical application of 4D-CT using 320-ADCT to evaluate the VPC function in pediatric patients with cleft palate. We also evaluated the organ-absorbed doses. Our method can be an alternative evaluation method for patients who refuse to undergo a conventional nasopharyngoscopic evaluation. We believe that further research will apply these merits to improve the quality of treatment and management of cleft patients.

\section{Abbreviations}

320-ADCT, 320-row area detector computed tomography; 3D, three-dimensional; 4D, four-dimensional; CSA, minimum cross-sectional area; CT, computed tomography; CTDIvol, volume of the CT dose index DICOM, Digital Imaging and Communications in Medicine; DLP, dose length product; DRL, diagnostic reference level; 
FOV, field of view; ICRP, International Commission on Radiological Protection; MC, Monte Carlo; MPR, multiplanar reconstruction; ROI, region of interest; SLP, speech-language pathologist; VPC, velopharyngeal closure; VPF, velopharyngeal function; VPI, Velopharyngeal insufficiency

\section{Declarations}

\section{Ethics approval and consent to participate}

The study was conducted in accordance with the Declaration of Helsinki (1964) and was approved by the Fujita Health University Ethics Review Committee (Aichi, Japan; reference number: HM18-431). All guardians of the participants were sufficiently informed about the purpose of this study, and they provided written informed consent.

\section{Consent for publication}

All parents or legal guardians of the participants gave written informed consent for their child's personal or clinical details, along with any identifying images, to be published in this study.

\section{Availability of data and materials}

The datasets supporting the conclusions of this article are available from the corresponding author on reasonable request.

\section{Competing interests}

The authors declare that they have no competing interests.

\section{Funding}

This work was supported by the Japanese Society for the Promotion of Science KAKENHI [grant number JP17K17299]. The funding sponsor had no role in the collection, analysis, or interpretation of the data or in the decision to submit the manuscript for publication.

\section{Authors' contributions}

YKB designed the study and wrote the initial draft of the manuscript. YKB, MK, and YKO carried out the dosimetry estimation. YKB and YS performed the computation. DK, NF, YKO, SY, and KS contributed to the design of the study and the analysis and interpretation of data and assisted in the preparation of the manuscript. HT, IT, and KM contributed to data collection and interpretation and critically reviewed the manuscript. All authors approved the final version of the manuscript and have agreed to be accountable for all aspects of the work in ensuring that questions related to the accuracy or integrity of any part of the work are appropriately investigated and resolved.

\section{Acknowledgements}

The authors would like to thank Professor Kensei Naito for contributing to the design of this study. 


\section{References}

1. Rajan S, Kurien M, Gupta AK, Mathews SS, Albert RR, Tychicus D. Velopharyngeal incompetence in patients with cleft palate, flexible video pharyngoscopy and perceptual speech assessment: a correlational pilot study. J Laryngol Otol. 2014;128:986-90.

2. Kobayashi Y, Kanamori D, Fujii N, Kataoka Y, Hirai E, Yoshioka S, et al. Velopharyngeal closure analysis using four-dimensional computed tomography: a pilot study of healthy volunteers and adult patients with cleft palate. BMC Med Imaging. 2019;19:54.

3. Sakamoto Y, Soga S, Jinzaki M, Yamada Y, Ogata H, Kishi K. Evaluation of velopharyngeal closure by 4D imaging using 320-detector-row computed tomography. J Plast Reconstr Aesthet Surg. 2015;68:47984.

4. Croft CB, Shprintzen RJ, Rakoff SJ. Patterns of velopharyngeal valving in normal and cleft palate subjects: a multi-view videofluoroscopic and nasendoscopic study. Laryngoscope. 1981;91:265-71.

5. Kobayashi M, Asada Y, Haba T, Matsunaga Y, Matsubara K, Minami K. Optimisation of swallowing CT examination: dose reduction and image quality. Radiat Prot Dosimetry. 2019;185:421-3.

6. Kanamori D, Kagaya H, Fujii N, Inamoto Y, Nakayama E, Suzuki S, et al. Examination of the distance measurement error and exposed dose when using a 320-row area detector CT: a comparison with videofluoroscopic examination of swallowing. Jpn J Compr Rehabil Sci. 2011;2:18-23.

7. Perry JL, Kuehn DP, Sutton BP, Fang X. Velopharyngeal structural and functional assessment of speech in young children using dynamic magnetic resonance imaging. Cleft Palate Craniofac J. 2017;54:40822.

8. Perry JI, Mason K, Sutton BP, Kuehn DP. Can dynamic MRI be used to accurately identify velopharyngeal closure patterns? Cleft Palate Craniofac J. 2018;55:499-507.

9. International Commission on Radiological Protection (ICRP). Report of the task group on reference man: ICRP Publication 23. Oxford: Pergamon Press; 1975.

10. Commission on Radiological Protection (ICRP). Radiological protection and safety in medicine: ICRP Publication 73. Oxford: Pergamon Press; 1996.

11. Vañó E, Miller DL, Martin CJ, Rehani MM, Kang K, Rosenstein M, et al. ICRP Publication 135: diagnostic reference levels in medical imaging. Ann ICRP. 2017;46:1-44.

12. Yonekura Y. Diagnostic reference levels based on latest surveys in Japan-Japan DRLs 2015. In: Japanese Network for Research and Information on Medical Exposures (J-RIME). http://www.radher.jp/J-RIME/report/DRLhoukokusyoEng.pdf. Accessed July 9, 2020.

\section{Figures}




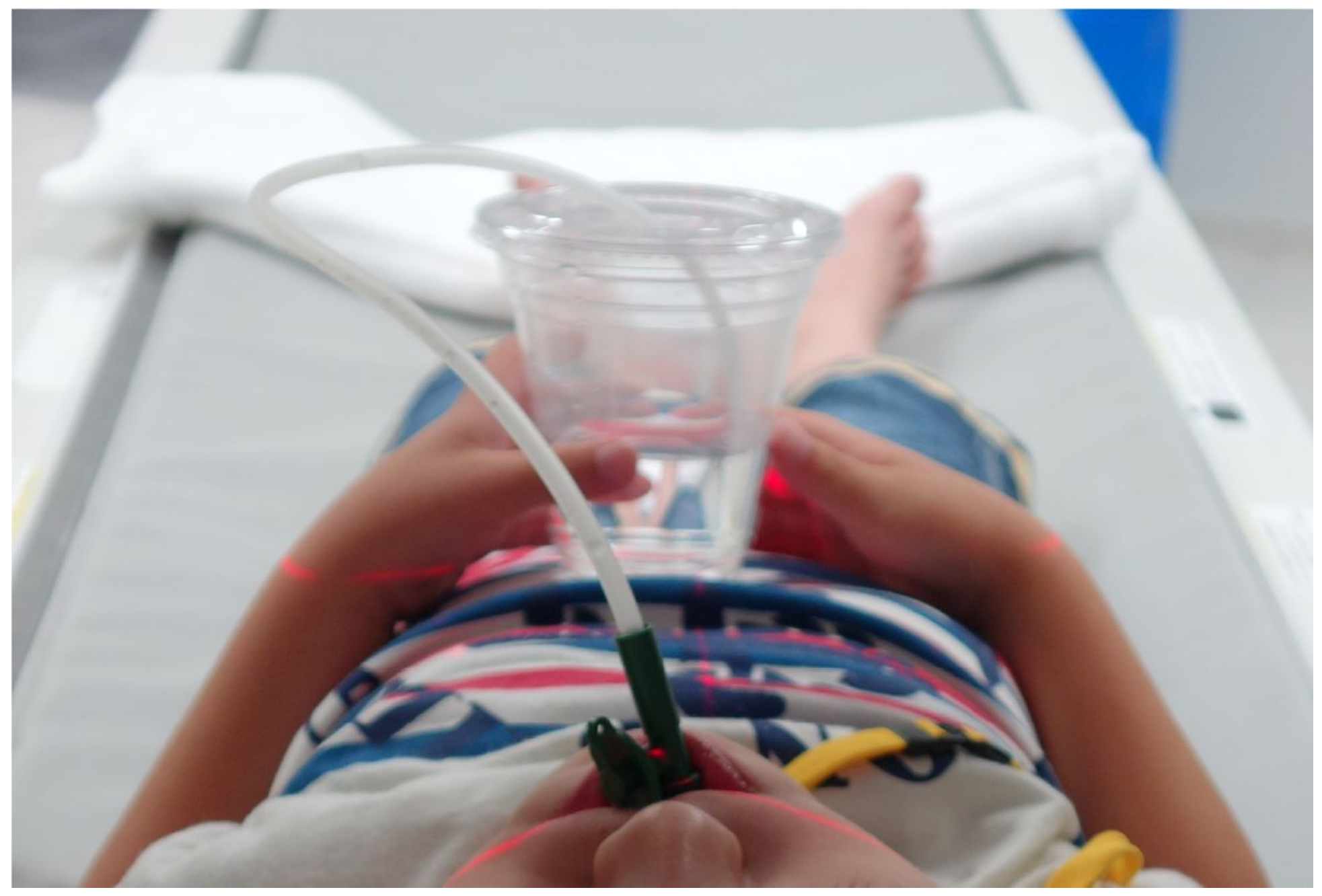

\section{Figure 1}

The test protocol. Each participant performed two tasks: nasal inspiration, followed by forceful expiration through a catheter into a water-filled cup. The parents of the participant gave written consent for the identifying images to be published in this study.
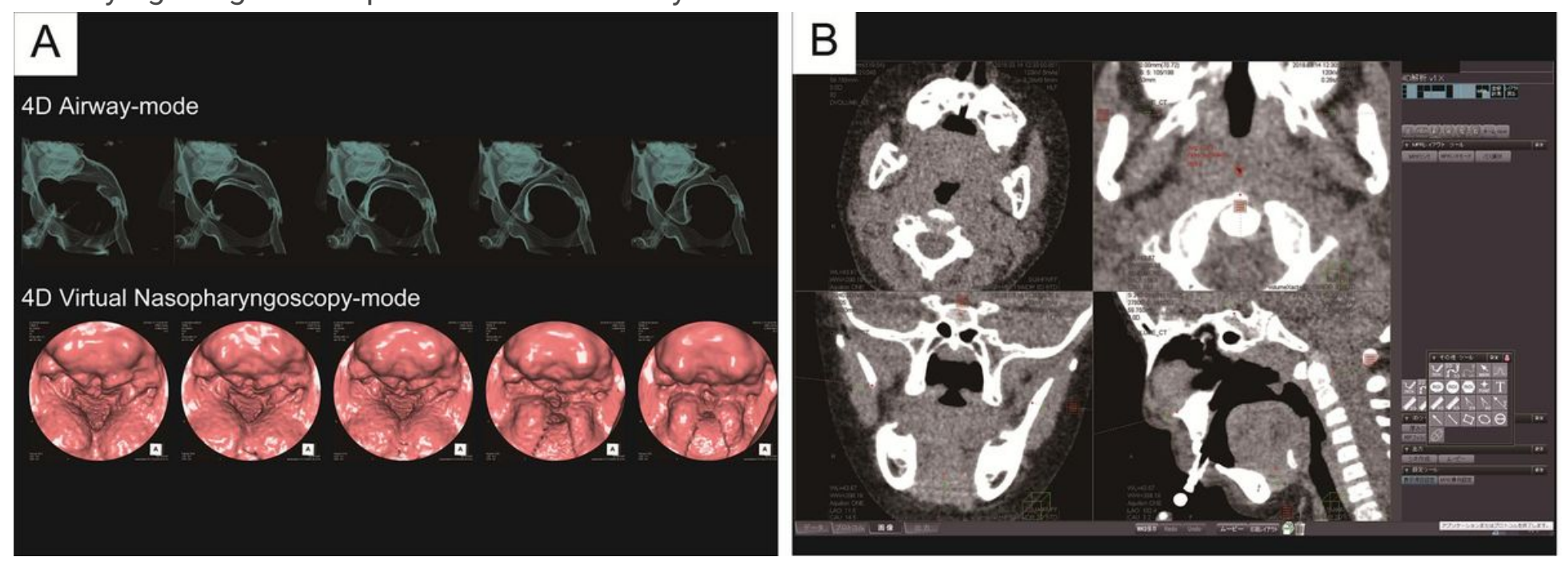

Figure 2 
Images of the airway in Patient \#2. a The four-dimensional (4D) mode (top) and 4D images of virtual nasopharyngoscopy-mode (bottom). b Multiplanar reconstruction images used to calculate the crosssectional area of velopharyngeal insufficiency.

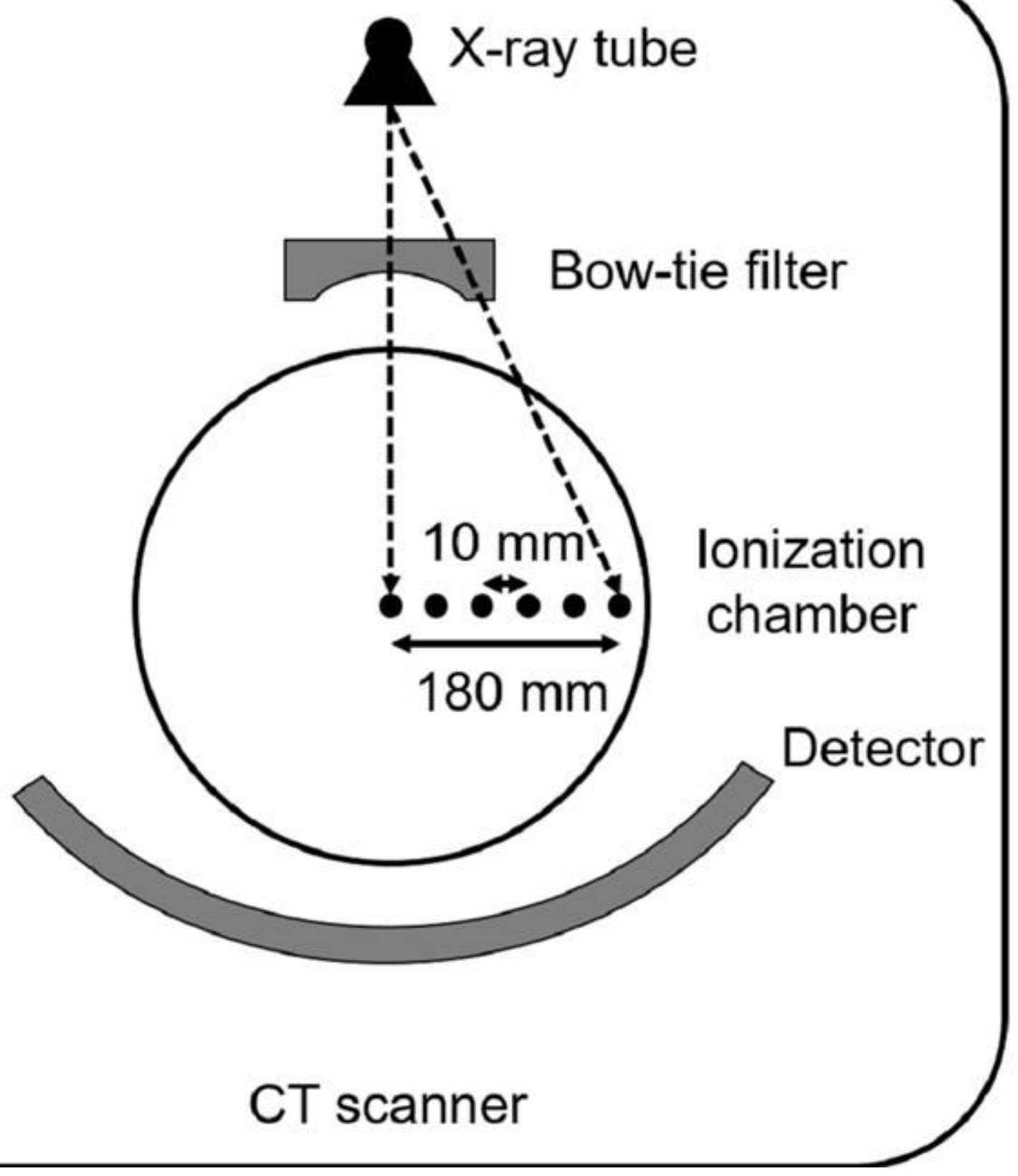

Figure 3

Schematics of velopharyngeal closure. The patterns of velopharyngeal closure are categorized into four groups, as described in previous literature [4]. (This figure is cited in our past report [2].) 


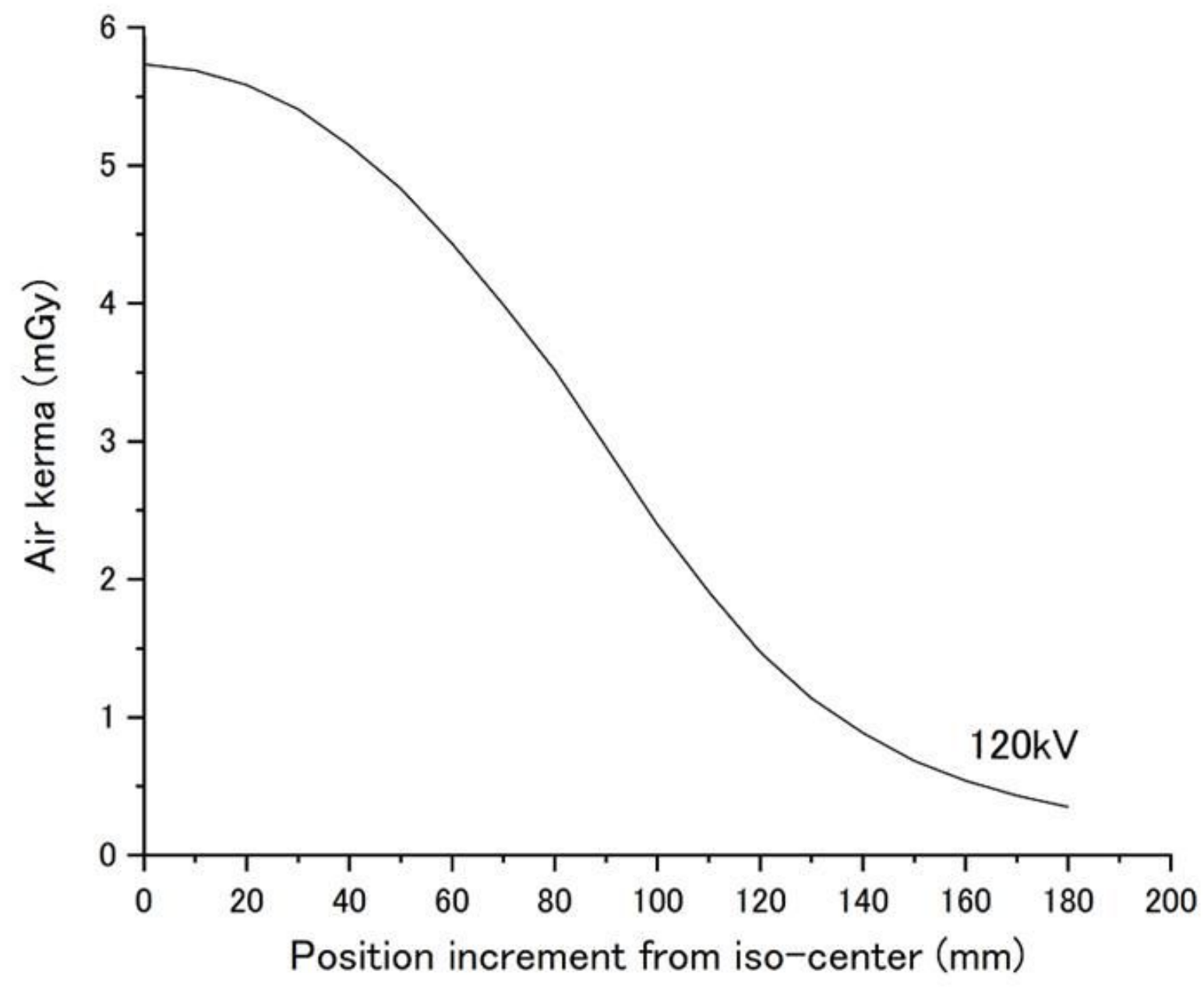

\section{Figure 4}

Protocol for the exposure dose calculation. a The bow-tie filter data are obtained by measuring along a straight line at a certain distance to the x-ray tube without rotation. $b$ The dose-rate curve. CT, computed tomography 


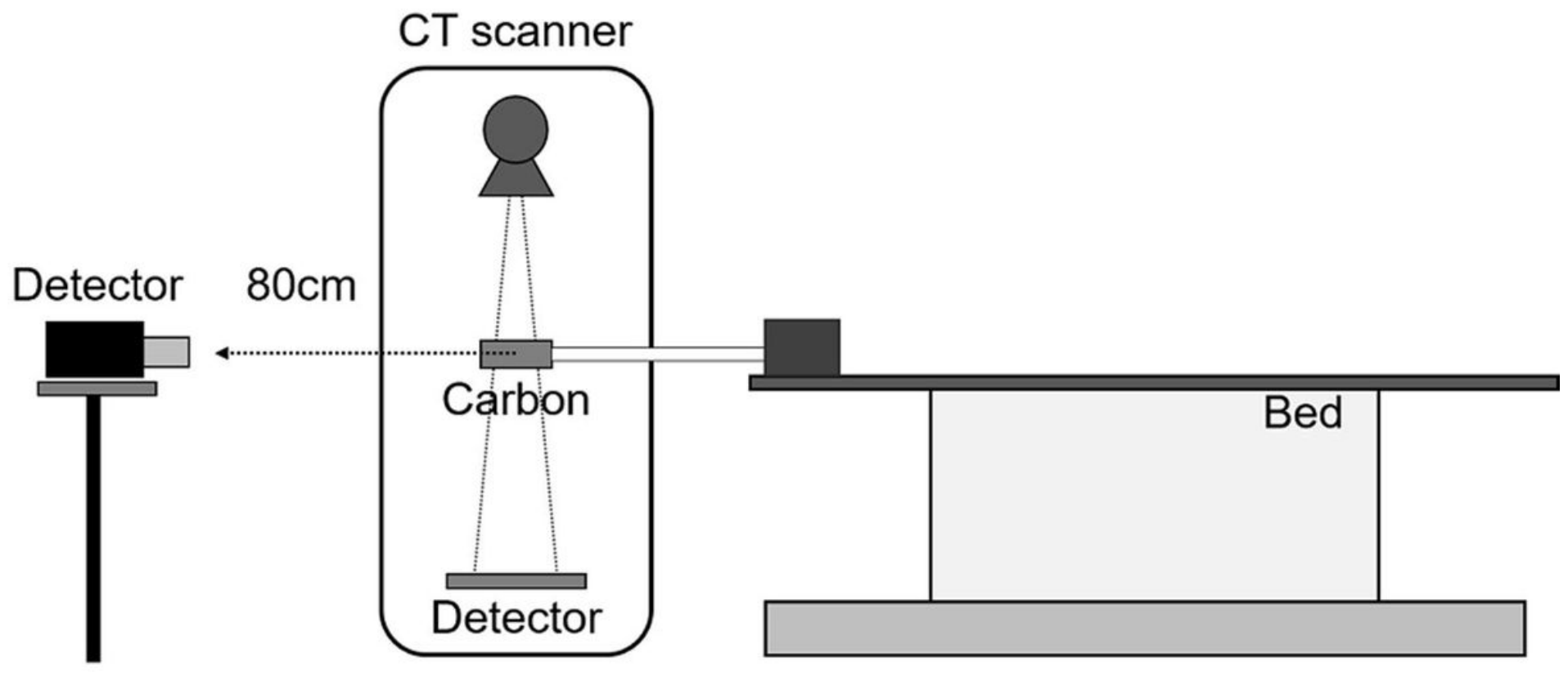

Figure 5

The schema indicates the measurement of the energy spectrum in the computed tomography (CT) scanner.

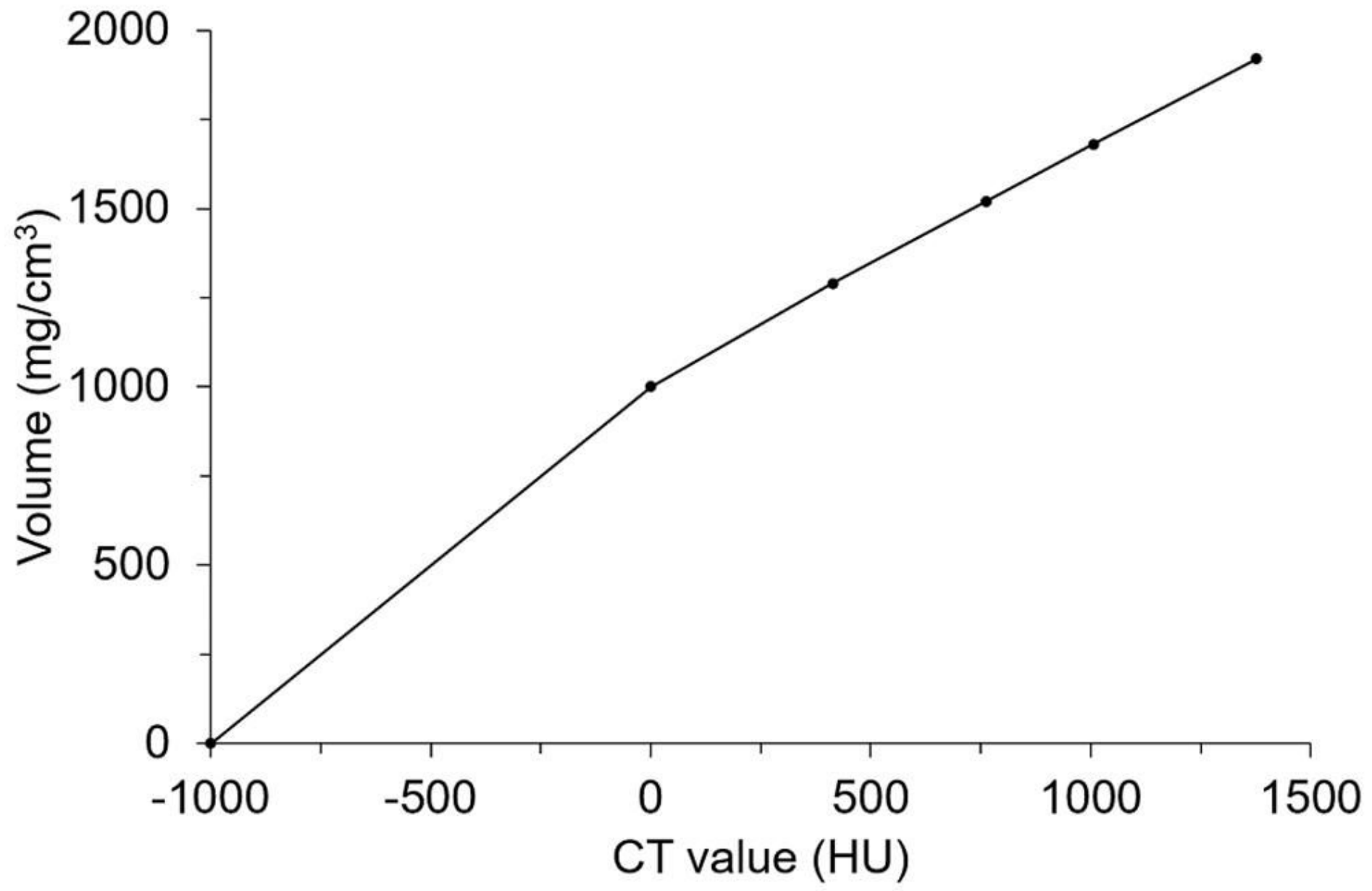

Figure 6 
Relationship between density and the computed tomography (CT) value in the Monte Carlo simulation.

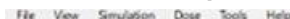

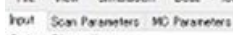

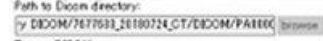

Tyse cosom

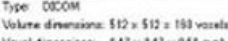

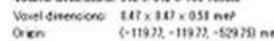

nowver ste if

Colurnitio at
slcelloc

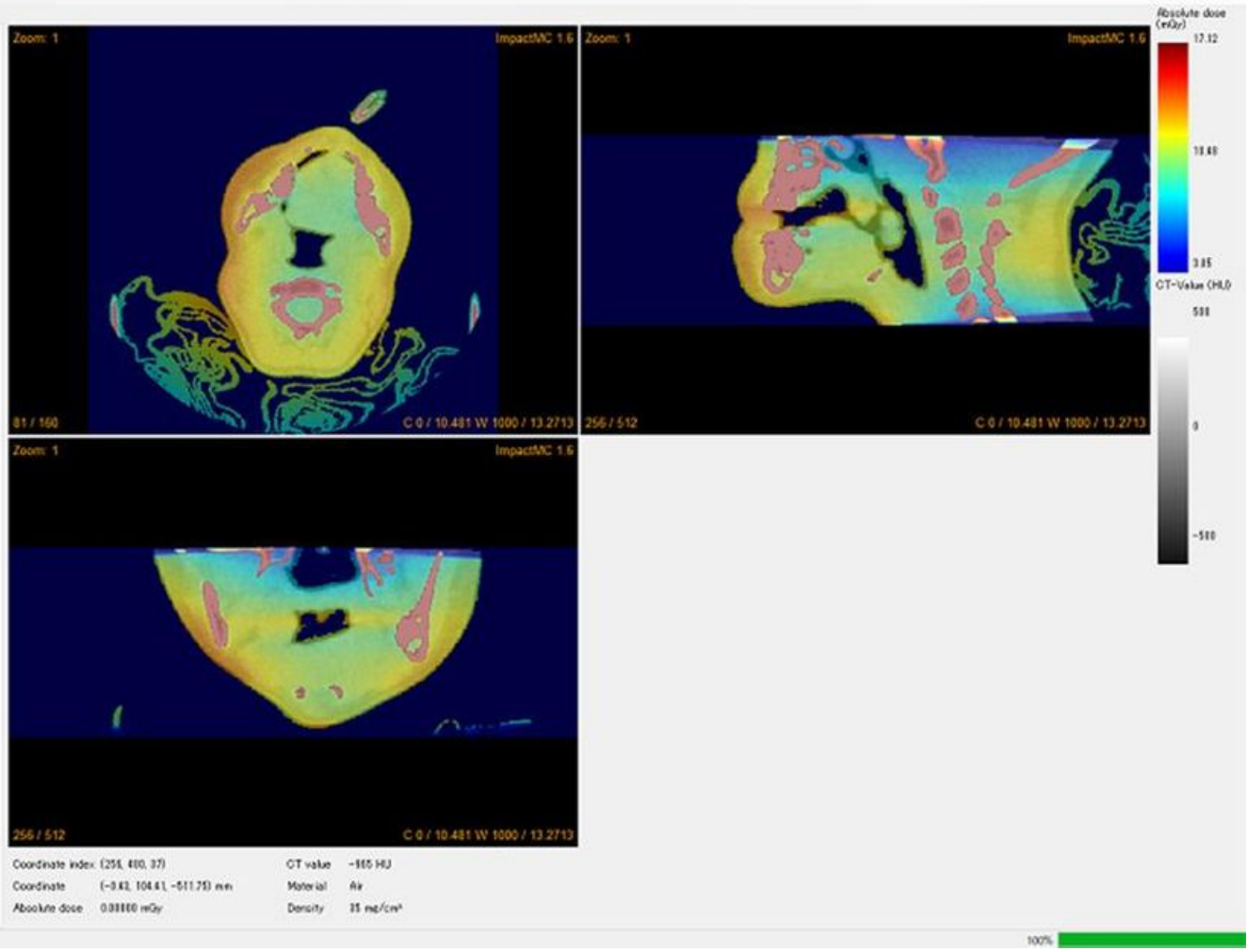

\section{Figure 7}

Distribution of the absorbed dose in the Monte Carlo simulation.
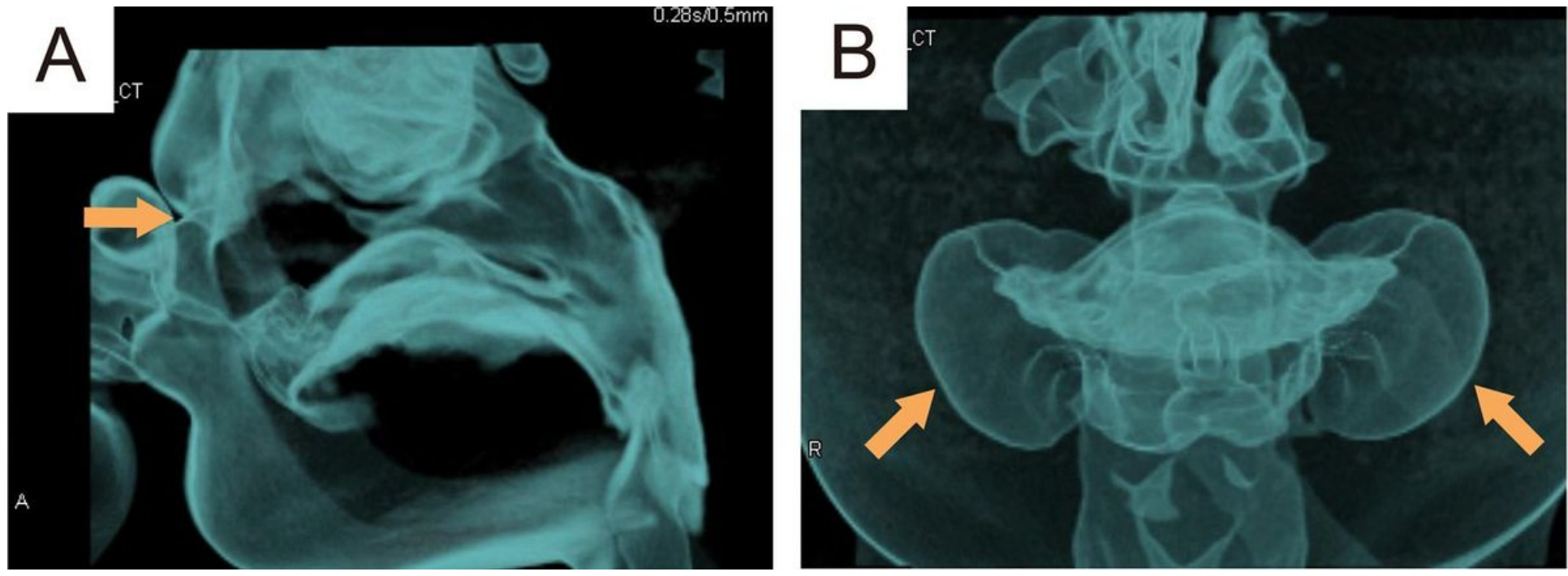

\section{Figure 8}

Some participants had irregular blowing. a Patient \#4 sealed the nostril with the upper lip (arrow). b Patient \#5 blew the air stored in the oral vestibule (arrow).

\section{Supplementary Files}


This is a list of supplementary files associated with this preprint. Click to download.

- Video.11.wmv 